\title{
O USO DO PROTETOR BUCAL NAS ARTES MARCIAIS: CONSCIÊNCIA E ATITUDE
}

\author{
MOUTHGUARD USE IN MARTIAL ARTS: AWARENESS AND ATTITUDE \\ EL USO DEL PROTECTOR BUCAL EN LAS ARTES MARCIALES: CONCIENCIA Y ACTITUD
}

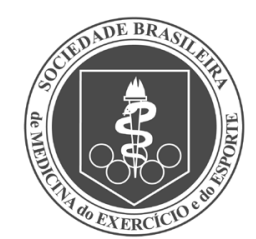

Artigo Original

\begin{abstract}
Camilla Cristina Lira Di Leone' (Cirurgiã-Dentista)

Igor Raphael Carvalho Nunes Barros' (Cirurgião-Dentista)

Alessandro Guimarães Salles²

(Cirurgião-Dentista)

Lívia Azeredo Alves Antunes

(Cirurgiã-Dentista)

Leonardo dos Santos Antunes (Cirurgião-Dentista)

1. Universidade Federal Fluminense, Faculdade de Odontologia, Pólo Universitário de Nova Friburgo, RJ, Brasil.

2. Universidade Federal Fluminense, Faculdade de Odontologia, Niterói, RJ, Brasil.
\end{abstract}

\section{Correspondência:}

Leonardo dos Santos Antunes, Rua Doutor Silvio Henrique Braune, 22 Centro, 28625-650 Nova Friburgo, RJ, Brasil. leonardoantunes@id.uff.br

\section{RESUMO}

Introdução: O uso de protetores bucais durante a prática de esportes de contato é de fundamental importância como medida preventiva dos traumatismos orofaciais. Objetivo: Realizar um levantamento quanto a utilização do protetor bucal entre praticantes de artes marciais, assim como verificar qual é a percepção que tais esportistas têm sobre sua importância e função. Métodos: Realizou-se uma pesquisa descritiva e transversal, de caráter investigativo, composta por 231 praticantes de três diferentes modalidades de artes marciais: Jiu-jítsu, Tae kwon do e Muay Thai, nas cidades de Nova Friburgo-RJ e Macaé-RJ, Brasil, por meio de um questionário estruturado. Os dados foram tabulados no programa estatístico SPSS 16.0. Foi realizada a frequência, sendo aplicado, ainda, o teste qui-quadrado $(p<0,05)$, para estabelecer relação entre variáveis de interesse. Resultados: Os dados indicaram que $60,6 \%$ dos entrevistados nunca receberam informações sobre o traumatismo orofacial durante a prática esportiva e que $46,3 \%$ dos esportistas já sofreram algum tipo de traumatismo orofacial. A maioria dos atletas $(93,9 \%)$ considera importante o uso do protetor bucal, porém este dado é desproporcional ao percentual de usuários do dispositivo: 78,7\% no Muay Thai, 60,9\% no Jiu-jitsu e 47,5\% no Tae kwon do ( $p<0,01)$. Dos protetores bucais existentes no mercado, o mais utilizado é o termoplástico ou tipo II (52,5\%) em todas as modalidades de lutas avaliadas. O protetor bucal personalizado causou menor percentual de interferência no rendimento durante a prática esportiva quando comparado com os outros tipos de protetores $(p<0,07)$. Conclusão: A maioria dos esportistas reconhece a importância do uso do protetor bucal, apesar de relatar não ter recebido informações sobre traumatismo orofacial durante a prática esportiva; a maioria dos atletas utiliza o protetor bucal termoplástico; há necessidade de se difundir informações sobre as vantagens do uso do protetor bucal personalizado, assim como, estimular o uso por parte de todos os praticantes de artes marciais.

Palavras-chave: prevenção de acidentes, traumatismos em atletas, esportes.

\section{ABSTRACT}

Introduction: The use of mouthguard during contact sports is crucial for preventing orofacial trauma. Objective: To conduct a survey regarding the use of mouthguards among martial arts practitioners as well as find what is the perception that these athletes have about its importance and function. Method: A descriptive cross-sectional survey of investigative character was conducted with 231 practitioners of three different kinds of martial arts (Brazilian Jiu-Jitsu, Muay Thai and Tae Kwon Do) in the cities of Nova Friburgo and Macaé, in Rio de Janeiro, Brazil, through a structured questionnaire. The data was analyzed in the SPSS 16.0 statistical software. The frequency was obtained and the Chi-square Test applied $(p<0.05)$ to establish association among variables of interest. Results: The data indicated that $60.6 \%$ of the respondents had never received information about orofacial trauma during sports practice and that $46.3 \%$ of the athletes have suffered some sort of orofacial injury. Most athletes (93.9\%) consider important to use the mouth guard, but this datum is disproportionate to the percentage of users of the device: $78.7 \%$ in Muay Thai, $60.9 \%$ in Jiu-Jitsu and $47.5 \%$ in Tae Kwon Do ( $p<0.01)$. Among the mouthguards on the market, the most widely used is the thermoplastic or type II (52.5\%) in all forms of wrestling evaluated. The custom mouthguard caused the lowest percentage of interference of performance during sports practice when compared with other protective devices ( $p<0.07)$. Conclusion: Most athletes recognize the importance of using a mouthguard, although they reported not having received information about orofacial trauma during sports practice; most athletes use thermoplastic mouthguard; it is necessary to disseminate information about the benefits of using custom mouthguard (type III) as well as encourage the use of this particular type by all practitioners of martial arts.

Keywords: accident prevention, athletic injuries, sports.

\section{RESUMEN}

Introducción: El uso de protectores bucales durante la práctica de deportes de contacto es de fundamental importancia como medida preventiva de los traumatismos orofaciales. Objetivo: Realizar un levantamiento sobre el uso del protector bucal entre practicantes de artes marciales, bien como verificar cuál es la percepción que tales deportistas tienen sobre su importancia y función. Métodos: Se realizó una encuesta descriptiva y transversal, de carácter investigativo, compuesta por 231 practicantes de tres diferentes modalidades de artes marciales: Jiu-jitsu, Tae kwon do y Muay Thai, en la ciudades de Nova Friburgo-RJ y Macaé-RJ, Brasil, por medio de un cuestionario 
estructurado. Los datos fueron tabulados en el programa estadístico SPSS 16.0. Fue realizada la frecuencia, siendo aplicado, además, el test chi-cuadrado $(p<0,05)$, para establecer relación entre variables de interés. Resultados: Los datos indicaron que 60,6\% de los entrevistados nunca recibieron informaciones sobre el traumatismo orofacial durante la práctica deportiva y que $46,3 \%$ de los deportistas ya sufrieron algún tipo de traumatismo orofacial. La mayoría de los atletas $(93,9 \%)$ considera importante el uso del protector bucal, aunque este dato es desproporcional al porcentual de usuarios del dispositivo: 78,7\% en Muay Thai, 60,9\% en Jiu-jitsu y 47,5\% en Tae kwon do ( $p<0,01)$. De los protectores bucales existentes en el mercado, el más utilizado es el termoplástico o tipo II (52,5\%) en todas las modalidades de lucha evaluadas. El protector bucal personalizado causó menor porcentual de interferencia en el rendimiento durante la práctica deportiva cuando comparado con los otros tipos de protectores $(p<0,07)$. Conclusión: La mayoría de los deportistas reconoce la importancia del uso del protector bucal, a pesar de relatar no haber recibido informaciones sobre traumatismo orofacial durante la práctica deportiva; la mayoría de los atletas utiliza el protector bucal termoplástico; hay necesidad de que se difundan informaciones sobre las ventajas del uso del protector bucal personalizado, asi como estimular el uso por parte de todos los practicantes de artes marciales.

Palabras clave: prevención de accidentes, traumatismos en atletas, deportes.

\section{INTRODUÇÃO}

O traumatismo orofacial, juntamente com a cárie, a doença periodontal e o câncer bucal pode ser considerado como um dos principais problemas de saúde pública'. Ao mesmo tempo em que atinge o indivíduo na sua integridade física, apresenta um agravante, a partir do momento em que interfere negativamente a nível psicológico ${ }^{2}$ podendo gerar impacto na qualidade de vida tanto do indivíduo quanto de sua família ${ }^{3}$.

Dentre as possíveis etiologias dos traumatismos orofaciais, destaca-se a prática esportiva ${ }^{4}$. Nos últimos anos, observou-se um crescimento do número de indivíduos praticantes de esportes em academias, clubes, quadras esportivas ou até mesmo ao ar livre. Desta forma, a tendência é de crescimento substancial no número de acidentes e traumatismos decorrentes de práticas esportivas?.

Segundo a "National Youth Sports Foundation", os atletas de esportes de contato têm cerca de 10\% a mais de possibilidade de sofrer lesões orofaciais durante uma competição esportiva, sendo de 33\% a 56\% durante toda a sua carreira. Dentre os esportes de contato, a prática de artes marciais oferece maior risco de ocasionar traumatismos dentários e fraturas, por ser mais frequente o contato com a face . $^{6}$

Os traumatismos orofaciais com origem na prática de esportes apresentam um grande diferencial: a possibilidade de se evitar ou pelo menos minimizar seus efeitos através do uso de dispositivos de proteção?

Através de uma meta-análise, Knapik et al. ${ }^{8}$ avaliaram a eficácia dos protetores bucais na redução das lesões. Os autores observaram que o risco de injúria era de 1,6 a 1,9 vezes maior quando os protetores bucais não foram usados durante a atividade esportiva. Neste contexto, a American Dental Association endossa o valor preventivo dos protetores bucais para uso dos esportistas em qualquer tipo de atividades recreativas com algum risco de lesão e em todos os níveis de competição?.

Dessa maneira, o presente estudo tem por objetivo realizar um levantamento quanto ao uso do protetor bucal entre praticantes de artes marciais nas cidades de Nova Friburgo e Macaé, no estado do Rio de Janeiro, Brasil, assim como verificar qual é a percepção que tais esportistas têm sobre a importância e função do uso destes dispositivos.

\section{MATERIAIS E MÉTODOS}

Este estudo foi submetido e aprovado pelo Comitê de Ética em Pesquisa do Hospital Universitário Antônio Pedro, da Universidade Federal Fluminense (Niterói, Rio de Janeiro, Brasil) sob o número 247/11. Sob o aspecto ético, uma explicação prévia foi feita aos praticantes de artes marciais para ser esclarecido o objetivo do estudo, a importância de sua execução para o meio científico e a importância de sua participação na mesma, com posterior assinatura do Termo de Consentimento Livre e Esclarecido (TCLE).

\section{Tipo de estudo}

Pesquisa transversal, de caráter investigativo, realizada com praticantes de artes marciais dos municípios de Nova Friburgo e Macaé localizados no estado do Rio de Janeiro, tendo em vista a avaliação quanto ao uso do protetor bucal por estes esportistas.

\section{Sujeito da pesquisa}

O grupo do estudo foi formado por 231 esportistas, entre profissionais e amadores, praticantes das artes marciais: Muay Thai, Jiu-jítsu e Tae kwon do.

Este estudo consistiu de uma amostragem de conveniência, uma vez que se caracterizou por não existir a preocupação com o desenho de um plano particular de amostragem. O objetivo não foi generalizar conclusões, pelo potencial viés de seleção, senão descrever as características principais do grupo do estudo ${ }^{10}$.

Como critério de inclusão, foram selecionados para a pesquisa esportistas, profissionais ou amadores, que praticavam as seguintes artes marciais: Muay Thai, Jiu-jítsu e Tae kwon do, ao menos uma vez por semana, sem limitações de idade.

Foram excluídos deste estudo os sujeitos que não autorizaram a participação no estudo, através da não assinatura do TCLE, questionários indevidamente preenchidos e atletas que praticassem outro tipo de arte marcial.

\section{MÉTODOS}

Inicialmente foi realizada uma seleção das academias nos Municípios de Nova Friburgo e Macaé, RJ, que tivessem em sua grade de aulas as artes marciais: Muay Thai, Jiu-jítsu e Tae kwon do. Em seguida os pesquisadores entraram em contato com os responsáveis obtendo consentimento para a realização da pesquisa.

Foi aplicado um questionário estruturado aos praticantes de artes marciais no período entre outubro de 2013 a março de 2014. O questionário foi pré-testado em 10 participantes com o objetivo de identificar problemas de entendimento nas perguntas que justificassem modificações da redação. Os questionários utilizados no pré-teste não foram incluídos na amostra final do estudo.

Para caracterização da amostra, foram incluídas perguntas a respeito da idade, gênero, nível econômico, grau de escolaridade, arte 
marcial praticada, o tempo e o número de vezes na semana. As outras perguntas foram direcionadas ao conhecimento dos praticantes de arte marcial sobre traumatismo orofacial, bem como sua ocorrência durante práticas esportivas. Também foi questionado quanto ao conhecimento, a importância e a utilização do protetor bucal, assim como o tipo de dispositivo utilizado, a dificuldade na sua utilização, interferência no seu rendimento e o sentimento de segurança.

\section{Análise estatística}

Para a análise quantitativa dos resultados, os dados foram tabulados no programa estatístico (SPSS 16.0) com digitação única, propiciando à pesquisa uma maior fidedignidade e confiabilidade. Utilizou-se a frequência como metodologia estatística para posterior discussão dos resultados. Foi aplicado, ainda, o teste qui-quadrado e t-Student em nível de $5 \%$ de significância $(p<0,05)$, para estabelecer relação entre variáveis de interesse.

\section{RESULTADOS}

Foram entrevistados 231 praticantes de artes marciais, sendo $162 \mathrm{em}$ Nova Friburgo-RJ e 69 em Macaé-RJ. Os dados relativos à média de idade, gênero, nível econômico e escolaridade encontram-se expressos na tabela 1, de acordo com o município que residiam.Em razão da homogeneidade da amostra e da ausência de diferença significativa entre os praticantes das duas cidades, optou-se por analisar os dados de forma conjunta.

Dos atletas entrevistados no presente estudo, 64 praticavam Jiu-jítsu, 40 Tae kwon do e 127 Muay thai. A maioria dos esportistas praticava a arte marcial a menos de um ano $(61,9 \%)$ e de três a cinco vezes na semana $(50,2 \%)$ sendo as distribuições percentuais bem aproximadas quando analisadas de acordo com o tipo de arte marcial praticada (tabela 2).

Na tabela 3, pode-se observar que apenas 39,4\% dos praticantes de artes marciais haviam recebido informações sobre traumatismo orofacial durante práticas esportivas, sendo que dos que receberam este tipo de informação, 60,4\% recebeu as orientações através das academias, 15,4\% pelo cirurgião-dentista e na faculdade e 24,2\% dividiu suas respostas entre em casa, televisão e amigos.

Tabela 1. Caracterização dos praticantes de artes marciais segundo o município dos entrevistados.

\begin{tabular}{|c|c|c|c|}
\hline & $\begin{array}{c}\text { Nova Friburgo } \\
\mathrm{N}=162\end{array}$ & Macaé $\mathrm{N}=69$ & P-valor \\
\hline Média de idade (DP) & $23,44( \pm 6,89)$ & $24,83( \pm 7,22)$ & $0,87^{*}$ \\
\hline \multicolumn{4}{|l|}{ Gênero (\%)* } \\
\hline Feminino & $38(23,5)$ & $15(21,7)$ & \multirow[b]{2}{*}{$0,776^{* *}$} \\
\hline Masculino & $124(76,5)$ & $54(78,3)$ & \\
\hline \multicolumn{4}{|l|}{ Nível econômico (\%) } \\
\hline$A 1-A 2$ & $17(10,5)$ & $14(20,3)$ & \multirow{4}{*}{$0,172^{* *}$} \\
\hline $\mathrm{B} 1-\mathrm{B} 2$ & $91(56,2)$ & $34(49,3)$ & \\
\hline $\mathrm{C} 1-\mathrm{C} 2$ & $52(32,1)$ & $19(27,5)$ & \\
\hline D & $02(1,2)$ & $02(2,9)$ & \\
\hline \multicolumn{4}{|l|}{ Grau de escolaridade (\%) } \\
\hline Analfabeto/Fundamental Incompleto & $3(1,9)$ & $0(0)$ & \multirow{5}{*}{ - } \\
\hline $\begin{array}{l}\text { Fundamental Completo/ } \\
\text { Fundamental Incompleto }\end{array}$ & $8(4,9)$ & $3(4,3)$ & \\
\hline $\begin{array}{l}\text { Fundamental } 2 \text { Completo/ } \\
\text { Médio Incompleto }\end{array}$ & $34(21)$ & $16(23,2)$ & \\
\hline Médio Completo/Superior Incompleto & $99(61,1)$ & $34(49,3)$ & \\
\hline Superior Completo & $18(11,1)$ & $16(23,2)$ & \\
\hline
\end{tabular}

Nota: * Teste $t$ Student; ** Teste do Qui-quadrado ou Exato de Fisher; valor * indica significância estatística $(p \leq 0.05)$
Tabela 2. Caracterização dos praticantes de artes marciais segundo as modalidades esportivas.

\begin{tabular}{c|c|c|c|c}
\hline Arte marcial & $\begin{array}{c}\text { Jiu-jítsu } \\
\mathrm{N}=64\end{array}$ & $\begin{array}{c}\text { Tae kwon } \\
\text { do } \mathrm{N}=40\end{array}$ & $\begin{array}{c}\text { Muay thai } \\
\mathrm{N}=127\end{array}$ & $\begin{array}{c}\text { Total } \\
\mathrm{N}=231\end{array}$ \\
\hline
\end{tabular}

Tempo que pratica arte marcial (\%)

\begin{tabular}{c|c|c|c|c}
\hline Até 1 ano & $31(48,4)$ & $29(72,5)$ & $83(65,3)$ & $143(61,9)$ \\
\hline Mais de 1 ano até 3 anos & $15(23,4)$ & $05(12,5)$ & $16(12,6)$ & $36(15,6)$ \\
\hline Mais de 3 anos até 5 anos & $07(10,9)$ & $02(5)$ & $09(7,1)$ & $18(7,8)$ \\
\hline Mais de 5 anos & $11(17,3)$ & $04(10)$ & $19(15)$ & $34(14,7)$ \\
\hline
\end{tabular}

Quantas vezes por semana pratica arte marcial (\%)

\begin{tabular}{c|c|c|c|c}
\hline Até 2 vezes na semana & $23(35,9)$ & $20(50)$ & $58(45,7)$ & $101(43,7)$ \\
\hline 3 a 5 vezes na semana & $37(57,8)$ & $19(47,5)$ & $60(47,2)$ & $116(50,2)$ \\
\hline Mais de 5 vezes na semana & $04(6,3)$ & $01(2,5)$ & $09(7,1)$ & $14(6,1)$ \\
\hline
\end{tabular}

Tabela 3. Aspectos relativos ao traumatismo orofacial e protetor bucal de acordo com as modalidades esportivas.

\begin{tabular}{c|c|c|c|c|c}
\hline Arte marcial & $\begin{array}{c}\text { Jiu-jítsu } \\
\mathrm{n}=64\end{array}$ & $\begin{array}{c}\text { Tae kwon } \\
\text { do } \mathrm{n}=40\end{array}$ & $\begin{array}{c}\text { Muay thai } \\
\mathrm{n}=127\end{array}$ & $\begin{array}{c}\text { Total } \\
\mathrm{n}=231\end{array}$ & $\mathrm{p}$-valor \\
\hline
\end{tabular}

Recebimento de informações de traumatismo orofacial durante práticas esportivas (\%)

\begin{tabular}{c|c|c|c|c|l}
\hline \multirow{2}{*}{$\operatorname{sim}$} & $23(35,9)$ & $25(62,5)$ & $43(33,9)$ & $91(39,4)$ & \multirow{2}{*}{$<0,01^{*}$} \\
\cline { 1 - 4 } Não & $41(64,1)$ & $15(37,5)$ & $84(66,1)$ & $140(60,6)$ & \\
\hline
\end{tabular}

Já sofreu traumatismo orofacial durante prática esportiva (\%)

\begin{tabular}{c|c|c|c|c|c}
\hline \multirow{2}{*}{$\operatorname{sim}$} & $35(54,7)$ & $20(50)$ & $52(40,9)$ & $107(46,3)$ & \multirow{2}{*}{0,17} \\
\cline { 1 - 4 } Não & $29(45,3)$ & $20(50)$ & $75(59,1)$ & $124(53,7)$ & \\
\hline
\end{tabular}

Conhecimento sobre o protetor bucal (\%)

\begin{tabular}{c|c|c|c|c|c}
\hline $\operatorname{sim}$ & $61(95,3)$ & $37(92,5)$ & $123(96,9)$ & $221(95,7)$ & \multirow{2}{*}{0,49} \\
\cline { 1 - 4 } Não & $3(4,7)$ & $3(7,5)$ & $4(3,1)$ & $10(4,3)$ & \\
\hline
\end{tabular}

Considera importante o uso do protetor bucal durante prática esportiva (\%)

\begin{tabular}{c|c|c|c|c|c}
\hline \multirow{2}{*}{$\operatorname{sim}$} & $60(93,8)$ & $35(87,5)$ & $122(96,1)$ & $217(93,9)$ & \multirow{2}{*}{0,14} \\
\cline { 1 - 4 } Não & $4(6,2)$ & $5(12,5)$ & $5(3,9)$ & $14(6,1)$ & \\
\hline
\end{tabular}

Utilização do protetor bucal (\%)

\begin{tabular}{c|c|c|c|c|c}
\hline \multirow{2}{*}{$\operatorname{sim}$} & $39(60,9)$ & $19(47,5)$ & $100(78,7)$ & $158(68,4)$ & \multirow{2}{*}{$<0,01^{*}$} \\
\cline { 1 - 4 } Não & $25(39,1)$ & $21(52,5)$ & $27(21,3)$ & $73(31,6)$ & \\
\hline
\end{tabular}

Tipo de protetor bucal (\%)

\begin{tabular}{c|c|c|c|c|c}
\hline Pré-fabricado & $10(25,6)$ & $07(36,8)$ & $23(23)$ & $40(25,3)$ & \\
\hline Termoplástico & $18(46,2)$ & $11(57,9)$ & $54(54)$ & $83(52,5)$ & \multirow{2}{*}{0,310} \\
\hline Personalizado & $11(28,2)$ & $01(5,3)$ & $23(23)$ & $35(22,2)$ & \\
\hline
\end{tabular}

Utilização do protetor bucal já preveniu traumatismo orofacial? (\%)

\begin{tabular}{c|c|c|c|c|c}
\hline $\operatorname{sim}$ & $30(76,9)$ & $14(73,7)$ & $69(69)$ & $113(71,5)$ & \multirow{2}{*}{0,633} \\
\cline { 1 - 4 } Não & $09(23,1)$ & $05(26,3)$ & $31(31)$ & $45(28,5)$ & \\
\hline
\end{tabular}

Nota: Teste do Qui-quadrado ou Exato de Fisher; valor *indica significância estatística $(p \leq 0.05)$. 
Nas perguntas relacionadas aos protetores bucais, 95,7\% relatou conhecer os protetores, 93,9\% considerou importante a sua utilização, no entanto, apenas $68,4 \%$ faz uso deste dispositivo durante os treinos e/ou competições. Observou-se relação entre a utilização do protetor bucal e o tipo de arte marcial praticada $(p<0,01)$. O protetor bucal foi mais utilizado por praticantes de Muay thai (78,7\%).

Com relação ao tipo de protetor bucal, o personalizado foi o que causou menor percentual de interferência no rendimento durante prática esportiva segundo relato dos praticantes de artes marciais $(p<0,07)$ (Tabela 4).

Tabela 4. Percepção dos praticantes de artes marciais sobre o protetor bucal de acordo com o tipo que utiliza.

\begin{tabular}{|c|c|c|c|c|}
\hline & $\begin{array}{l}\text { Pré-fabricado } \\
\qquad \mathrm{N}=40\end{array}$ & $\begin{array}{c}\text { Termoplastificado } \\
\mathrm{N}=83\end{array}$ & $\begin{array}{l}\text { Personalizado } \\
\qquad \mathrm{N}=35\end{array}$ & P-Valor \\
\hline \multicolumn{5}{|c|}{ Dificuldade na adaptação / utilização do protetor bucal (\%) } \\
\hline Sim & $32(80)$ & $65(78,3)$ & $29(82,9)$ & \multirow{2}{*}{0,853} \\
\hline Não & $8(20)$ & $18(21,7)$ & $6(17,1)$ & \\
\hline
\end{tabular}

Sentimento de segurança durante prática esportiva (\%)

\begin{tabular}{c|c|c|c|c}
\hline $\operatorname{sim}$ & $38(95)$ & $81(97,6)$ & $35(100)$ & \multirow{2}{*}{0,386} \\
\cline { 1 - 3 } Não & $2(5)$ & $2(2,4)$ & $0(0)$ & \\
\hline
\end{tabular}

Interferência no seu rendimento durante prática esportiva (\%)

\begin{tabular}{c|c|c|c|c}
\hline $\operatorname{sim}$ & $10(25)$ & $23(27,7)$ & $3(8,6)$ & \multirow{2}{*}{0,07} \\
\cline { 1 - 3 } Não & $30(75)$ & $60(72,3)$ & $32(91,4)$ & \\
\hline
\end{tabular}

Nota: Teste do Qui-quadrado ou Exato de Fisher; valor significância estatística ( $p \leq 0.05)$.

\section{DISCUSSÃO}

A sociedade atualmente está vivenciando grandes mudanças em seu estilo de vida, incluindo cada vez mais hábitos saudáveis em sua rotina. Em busca de uma melhora na qualidade de vida, observa-se o crescimento do número de pessoas nas práticas esportivas. Porém, sabe-se que a prática de esportes é um dos principais fatores responsáveis pelos altos índices de lesões orofaciais, por ser uma atividade na qual os atletas estão mais sujeitos a sofrer um possível trauma, podendo influenciar no seu desempenho e na sua qualidade de vida.

Com o intuito de minimizar as sequelas produzidas por esses acidentes, observa-se a necessidade, por parte dos cirurgiões-dentistas, de lançar mão de medidas de prevenção, entre elas o protetor bucal. Contudo, existem poucos levantamentos epidemiológicos acerca do conhecimento relacionado ao traumatismo orofacial e a utilização do protetor bucal como método de proteção entre esportistas ${ }^{11}$.

Diversos trabalhos indicam que os índices de traumatismos em esportes variam de acordo com o esporte praticado, ${ }^{11,12}$ sendo esta uma das razões para ter sido desenvolvido o presente estudo em praticantes de artes marciais.

No presente estudo pôde ser observada a presença de uma grande deficiência no recebimento de informações a respeito de traumatismo orofacial por parte dos praticantes de artes marciais, resultado compatível com o apresentado pela literatura ${ }^{11,12}$. Dos praticantes de artes marciais que haviam recebido orientações, apenas 15,4\% havia recebido as mesmas de cirurgiões-dentistas e nas faculdades, sendo assim, sugere-se que cabe aos odontólogos uma maior atenção ao assunto, abordando-o dentro de programas de prevenção e promoção à saúde bucal, com o objetivo de informar, conscientizar e promover o uso correto dos protetores bucais junto a todos os praticantes de esporte.
Sane e Ylipaavalniemi ${ }^{4}$ e Ferrari et al. ${ }^{11}$ relatam que a ocorrência de traumas dentários durante as práticas esportivas apresenta altos índices, essa afirmação é confirmada na presente pesquisa, uma vez que 46,3\% dos entrevistados já haviam sofrido traumatismo orofacial durante a prática esportiva. Este resultado foi alto se comparado ao estudo realizado por Ferrari, Simi e Medeiros ${ }^{11}$ sobre a ocorrência de traumatismo dental em atletas de diferentes modalidades de esportes de contato, onde apenas 28,8\% haviam relatado essa situação. Esta diferença pode ser explicada, uma vez que os atletas no presente estudo praticam somente artes marciais e esse tipo de prática está mais susceptível ao traumatismo orofacial, pois os praticantes têm um maior contato físico do que o grupo pesquisado por Ferrari, Simi e Medeiros ${ }^{11}$, os quais mesmo envolvendo artes marciais como judô e jiu-jítsu, também envolveram em seus resultados outras práticas esportivas, tais como, hóquei, futebol, basquete e handebol.

Uma maneira de minimizar o trauma orofacial seria adotar o uso de protetores bucais em esportes de contato físico. Na presente pesquisa, $71,5 \%$ dos praticantes de artes marciais relataram que tal dispositivo já preveniu a ocorrência de traumatismos orofaciais. Segundo Knapik et al. ${ }^{8}$, o aparelho tem o poder de distribuir as forças durante o golpe, minimizando os efeitos do impacto sobre os tecidos e, dessa forma, diminuindo a quantidade e a gravidade dos danos causados.

Diferentemente do encontrado nas pesquisas de Barberine et al., ${ }^{13}$ Bastida et al. ${ }^{12}$ e Ferrari et al. ${ }_{.1}{ }^{11}$ onde 40\%, 34,6\% e 15,9\%, respectivamente, fazem uso do protetor bucal, esta pesquisa observou a maioria dos praticantes fazendo uso do dispositivo durante a prática esportiva. Sugere-se que esse resultado tenha sido modificado atualmente por influência da ação da mídia, onde as artes marciais vem ganhando um grande espaço através de lutas televisionadas em canais abertos, em virtude do sucesso de atletas profissionais brasileiros nas modalidades de MMA (Mixed Martial Arts).

Quase que a totalidade dos esportistas (97,5\%) sentiu-se segura com a utilização do protetor, o que denota a grande receptividade desses atletas à implementação do uso do aparelho. A American Society for Testing and Materials, normatizou os protetores bucais como sendo divididos em três categorias (ASTM, 2006). ${ }^{14}$ Os pré-Fabricados (Estoque), são os que possuem menor custo, baixa efetividade, desconforto em virtude do maior volume e deficiência na adaptação; ocasionando dificuldade na fala e na respiração. Os Termoplásticos (boil-and-bite), aqueles confeccionados a partir de uma moldeira termoplástica pré-conformada, de tamanho padrão, modelada na boca. Os Individuais (personalizados), confeccionados pelo cirurgião-dentista, oferecem uma adequada adaptação, retenção e melhor proteção, além de proporcionar um maior conforto ao usuário; não interferindo na fonação, respiração e na ingestão de líquidos. Embora os atletas entrevistados tenham consciência da importância do uso do dispositivo, a maioria utilizava o protetor termoplástico ou tipo II. Concordando com Bastida et al., ${ }^{12}$ acredita-se que o principal motivo para aquisição destes protetores seja o fácil acesso, uma vez que são vendidos em lojas de artigos de esportes e farmácias, além do baixo custo e a simplicidade da técnica para confecção. É preciso expandir as orientações da melhor forma de utilização do aparelho, indicando o protetor bucal que desempenha todas as características necessárias para uma correta prevenção, ou seja, o protetor bucal individualizado ou tipo III.

A maioria dos praticantes de artes marciais revelou que tiveram dificuldade na adaptação e utilização do protetor bucal. Por isso sugere-se, conforme Canto et al., ${ }^{15}$ que a utilização deste utensílio deva ser iniciada cedo, para que, ainda criança, o esportista se habitue mais facilmente ao uso do dispositivo. É também importante que cirurgiões-dentistas assumam o papel de educadores junto aos pais, responsáveis 
e treinadores, orientando sobre os riscos de acidentes durante a prática esportiva e a importância do uso do protetor bucal.

Uma vez que a atividade esportiva é um fator predisponente ao traumatismo e estando os atletas inevitavelmente expostos aos riscos de acidentes, a inclusão de programas educativo-preventivos junto a estes é imprescindível em todas as regiões do país. Cabe ao cirurgião-dentista tornar-se o responsável por orientar e incentivar os esportistas a uma prática segura e, de certa forma, a população em geral, acerca da importância de medidas preventivas relacionadas ao traumatismo orofacial.

Com base nos resultados encontrados, este estudo servirá de subsídio para elaboração de prioridades de ação preventiva, a fim de se buscar uma melhora na qualidade de vida de praticantes de artes marciais.

\section{CONCLUSÃO}

Com base nos resultados apresentados, pode-se concluir que a maioria dos esportistas reconhece a importância do uso do protetor bucal, apesar de relatarem não ter recebido informações sobre traumatismo orofacial durante a prática esportiva; a maioria dos atletas utiliza o protetor bucal termoplástico; há necessidade de se difundir informações sobre as vantagens do uso do protetor bucal personalizado, assim como estimular o uso por parte de todos os praticantes de artes marciais.

Todos os autores declararam não haver qualquer potencial conflito de interesses referente a este artigo.

\section{REFERÊNCIAS}

1. Peterson PE, Bourgeios D, Ogawa H, Estupidan-day S; Ndiyae C. The global burden of oral diseases and risks to oral health. Bull World Health Organ. 2005;83(9):661-9.

2. Silveira EG, Araujo SM, Schmitt BHE, Farias MMAG, Campos I; Caregnato M. Conhecimento e atitudes dos odontopediatras do estado de Santa Catarina acerca de mecanismos de prevenção de traumatismos bucais relacionados a esportes. Rev Odontol UNESP. 2009;38(6):341-6.

3. Antunes LA, Antunes LS, Luiz RR, Leão AT, Maia LC. Assessing the responsiveness of the Brazilian FIS to treatment for traumatic dental injury. Community Dent Oral Epidemiol. 2013;41(6):551-7.

4. Sane J, Ylipaavalniemi P. Dental trauma in contact team sports. Endod Dent Traumatol.1988:4(4):164-9.

5. Nogueira AS, Freire FFWV. Aspectos epidemiológicos e preventivos relacionados aos traumatismos orofaciais decorrentes de prática esportiva. In: Lima DLF. Odontologia esportiva: O cirurgião-dentista no cuidado do esportista. Editora Santos. Grupo Gen. 2013. p. 75-92.

6. Santiago E, Simões R, Soares D, Pereira JA, Caldas T. Protetor bucal "custom-made": indicações, confecção e características essenciais. Arq. Med. 2008;22(1):25-33.

7. Perkins SW, Dayan SH, Sklarew EC, Hamilton M, Bussell GS. The incidence of sports-related facial trauma in children. Ear Nose Throat J. 2000;79(8):632-4.
8. Knapik JJ, Marshall SW, Lee RB, et al. Mouthguards in sport activities: history, physical properties and injury prevention effectiveness. Sports Med. 2007; 37(2):117-44.

9. American Dental Association. Policy Statement on Orofacial Protectors. Transactions; 1995. p. 613.

10. Luiz R, Tânia G, Magnanini M. Planejamento amostral. In: Luiz R, Costa A, Nadanovsky P. Epidemiologia e bioestatística na pesquisa odontológica. São Paulo: Atheneu; 2005. p. 245-72.

11. Ferrari $\mathrm{CH}$, Simi J. J, Medeiros JMF de. Ocorrência de traumatismo dental e nível de esclarecimento e uso do protetor bucal em diferentes grupos de esportistas. Avaliable from: http://www.odontologia.com. br/artigos.asp?id=88\&i desp=2\&ler=s, 2000 Mar. Medcenter.com Odontologia, São Paulo, 2000, p. 1-5.

12. Bastida EM, Peron RAF, Queiroz AF, Hayacibara MF, Terada RSS. Prevalência do uso de protetores bucais em praticantes de artes marciais de um município do Paraná. Rev Bras Odontol. 2010;67(2): 194-8.

13. Barberini AF, Aun CE, Caldeira CL. Incidência de injúrias orofaciais e utilização de protetores bucais em diversos esportes de contato. Rev Odontol UNICID. 2002;14(1):7-14.

14. American Society for Testing and Materials. Standard practice for care and use of athletic mouth protectors. ASTM F697-00. Philadelphia, Pa: American Society for Testing and Materials; Reapproved 2006

15. Canto G.L, Oliveira J, Hayasaki S.M, Cardoso M. Protetores bucais: uma necessidade dos novos tempos. Rev Dent Press Ortodon Ortop Facial. 1999;(4)6:20-6 